\title{
HABILIDADES BLANDAS Y SU IMPACTO EN LA GESTIÓN DE LA MEZCLA DE MARKETING. UN ESTUDIO REALIZADO EN EL EMPORIO COMERCIAL GAMARRA, PERÚ.
}

\section{SOFT SKILLS AND ITS IMPACT ON THE MANAGEMENT OF THE MARKETING MIX. A STUDY CARRIED OUT IN GAMARRA COMMERCIAL EMPORIUM, PERU.}

Víctor Hugo Fernández Bedoya, Mgs. Magíster en Costos y Presupuestos (Perú). Docente en la Universidad César Vallejo, filial Lima Norte y Docente en la Universidad San Andrés, Perú.

victorhugofernandezbedoya@gmail.com

Ambrocio Teodoro Esteves Pairazamán, Ph.D. Doctor en Adminitración de la Educación (Perú). Docente en la Universidad César Vallejo, filial Lima Norte, Perú. diosteama774@hotmail.com

Janny Elsa Durand Peña, Mgs. Magíster en Administración y Dirección de Empresas (Perú). Docente en la Universidad César Vallejo, filial Lima Norte, Perú. jannydurand@gmail.com Henry Paul Núñez Soto, MBA. Magister en Administración de Negocios (Perú). Docente en la Universidad César Vallejo, filial Lima Norte, Perú. henrypaulns29@gmail.com

\section{ARTÍCULO DE INVESTIGACIÓN}

Recibido: 20 de diciembre de 2018.

Aceptado: 27 de marzo de 2019.

\section{RESUMEN}

En la actualidad, el factor humano que compone cada empresa puede llegar a ser considerado una ventaja competitiva, logrando diferenciar a ciertas compañías de otras, colocándole en una posición relativa superior para competir. Este estudio tiene como objetivo general: determinar en qué medida las habilidades blandas impactan en la 
gestión de la mezcla de marketing de las empresas del Emporio Comercial Gamarra, Perú. La hipótesis general es: las habilidades blandas impactan en la gestión de la mezcla de marketing de las empresas del Emporio Comercial Gamarra, Perú. El enfoque de la investigación es cualitativo, nivel correlativo-causal con diseño no experimental. La población es de 40928 personas, cuya muestra de 381 individuos nos permiten tener un $95 \%$ de confianza y $5 \%$ de error máximo. Se aplicó una encuesta a la muestra seleccionada, cuyos datos fueron procesados a través del programa SPSS. La hipótesis general y ocho hipótesis específicas fueron contrastadas y validadas mediante la prueba de Chi-cuadrado de Pearson, obteniendo para todas las hipótesis planteadas significación asintótica bilateral menor a 0,05, lo cual nos permite afirmar que existe asociación entre las variables sometidas y por ende aceptar dichas hipótesis. Se concluye que las habilidades blandas impactan positivamente en la gestión de la mezcla de marketing.

Palabras clave: habilidades blandas, mezcla de marketing, gestión, Emporio Comercial Gamarra.

\section{ABSTRACT}

In these days, the human factor that makes up each company can be considered as a competitive advantage, managing to differentiate certain companies from others, placing them in a higher relative position to compete. The general objective of this study is to determine the extent to which soft skills have an impact on the management of the marketing mix of companies in Gamarra Commercial Emporium, Peru. The general hypothesis is: soft skills have an impact on the management of the marketing mix of companies in the Gamarra Commercial Emporium, Peru. The focus of the research is qualitative, correlative-causal level with non-experimental design. The population is 40,928 people, whose sample is 381 people and that allow us to have a $95 \%$ confidence and a $5 \%$ maximum error. The general hypothesis and eight specific hypotheses are contrasted and validated by the Pearson Chi-square test, obtaining for all the hypotheses proposed bilateral asymptotic results less than 0.05 , which allows us to affirm that there is an association between the variables submitted and to accept these hypotheses. It is concluded that soft skills have a positive impact on the management of the marketing mix.

Keyword: soft skills, marketing mix, management, Gamarra Commercial Emporium. 


\section{INTRODUCCIÓN}

Hasta hace algunos años atrás, era común que todo empresario piense firmemente que para que una empresa lidere el mercado bastaba con que el personal del área de marketing tenga un buen manejo técnico de la mezcla de marketing tradicional, esto es, prestar atención a las condiciones del producto, del precio, de la plaza y de la promoción; aduciendo que será responsabilidad del área de ventas el asegurar que se cumpla el plan de ventas asignado.; sin embargo, el mundo empresarial ha cambiado, y con ello las empresas se han visto en la necesidad de contar con personalidad con habilidades que van más allá de conocimientos técnicos, se busca de integrantes con habilidades blandas como trabajo en equipo, liderazgo, comunicación y administración del tiempo.

El concepto de la mezcla de marketing ha atraído la atención tanto de académicos como de gerentes y ha sido ampliamente utilizado en la disciplina de marketing a nivel mundial (Plomaritou, 2008). Desde sus inicios hasta la actualidad, la mezcla de marketing ha tenido variaciones, sin embargo, es generalmente aceptado aplicar el modelo de las 4P tradicional defendida por McCarthy en 1960 para productos físicos, y el modelo de 7P definido por Booms y Bitner en 1981 para el caso de productos intangibles; cabe resaltar que muchos investigadores, profesores y profesionales del marketing suelen agregar otras "P" a la mezcla original (Jackson \& Ahuja, 2016). En esta investigación tendrá como variables de la mezcla de marketing las $4 \mathrm{P}$ tradicionales, siendo estas el producto, el precio, la plaza y la promoción.

Por otro lado, de acuerdo al último estudio de Linkedln sobre habilidades blandas, elaborado por Patrone (2018), y donde se analizaron los resultados de cuestionarios aplicados a 2000 líderes de negocios a nivel mundial, se concluyó que las cuatro habilidades blandas que se requieren a nivel empresarial, en general, son liderazgo, habilidades de comunicación, predisposición a la colaboración y administración del tiempo; cabe resaltar que estas cuatro habilidades blandas más solicitadas en el mundo pueden estar alineadas al cumplimiento de objetivos no abarcados por el marketing. Para este estudio se seleccionaron las dimensiones "liderazgo" y "habilidades de comunicación".

A pesar de que existen gran cantidad de investigaciones referentes a las habilidades blandas, muchas de estas miden el efecto de su desarrollo durante la etapa de educación, ya sea en la etapa escolar (Ortega, Febles y Estrada (2016), pregrado (Ascón, García y Pedraza, 2018; Merrero, Mohamed y Xifra, 2018), e inclusive posgrado 
(Zermeño y Lozano, 2016), por nombrar algunas de ellas, sin embargo, la gran mayoría no relacionan a las habilidades blandas con alguna variable asociada al marketing.

Además, es importante recalcar que el entorno empresarial está en constante cambio, por lo que las compañías deben de adoptar frecuentemente medidas relacionadas a la mejora continua (Minaya y Fernández, 2018), de esta manera, se asegurará no solo su supervivencia, sino el liderazgo del sector.

Visto esto, esta investigación pretende hallar el grado de impacto de las habilidades blandas en la mezcla de marketing, en empresas del Emporio Comercial Gamarra, ubicado en Perú, a fin de obtener información importante que permita medir su nivel de asociación y evaluar su pertinencia en cuanto a su implementación. Ante ello, se presenta el siguiente modelo teórico a modo de planteamiento del problema.

Figura 1. Modelo teórico para la investigación "habilidades blandas y su impacto en la gestión de la mezcla de marqueting de empresas del Emporio Comercial Gamarra, Perú"

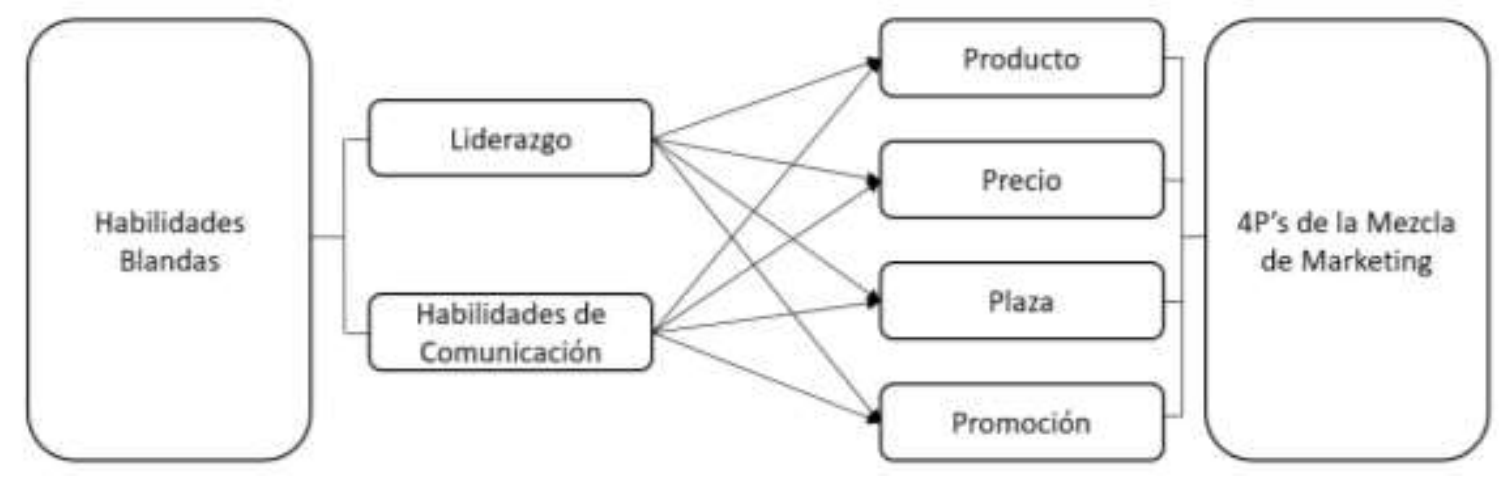

Fuente: Elaboración propia.

De acuerdo con Hernández Sampieri, Fernández y Baptista (2014), todo problema a investigar deberá formularse a través de la aplicación de preguntas directas a fin de reducir posibles distorsiones y para que de esta manera el investigador obtenga resultados coherentes para la investigación. Líneas abajo pueden apreciarse los problemas planteados:

Problema principal:

¿Las habilidades blandas impactan en la gestión de la mezcla de marketing de las empresas del Emporio Comercial Gamarra, Perú? 
Problemas secundarios:

¿El liderazgo impacta en la gestión del producto de las empresas del Emporio Comercial Gamarra, Perú?

¿El liderazgo impacta en la gestión del precio de las empresas del Emporio Comercial Gamarra, Perú?

¿El liderazgo impacta en la gestión de la plaza de las empresas del Emporio Comercial Gamarra, Perú?

¿El liderazgo impacta en la gestión de la promoción de las empresas del Emporio Comercial Gamarra, Perú?

¿Las habilidades de comunicación impactan en la gestión del producto de las empresas del Emporio Comercial Gamarra, Perú?

¿Las habilidades de comunicación impactan en la gestión del precio de las empresas del Emporio Comercial Gamarra, Perú?

¿Las habilidades de comunicación impactan en la gestión de la plaza de las empresas del Emporio Comercial Gamarra, Perú?

¿Las habilidades de comunicación impactan en la gestión de la promoción de las empresas del Emporio Comercial Gamarra, Perú?

Asimismo, y también con relación a lo detallado por Hernández Sampieri et al. (2014, p. 108), las hipótesis de tipo correlacional sirven para especificar las relaciones entre más de una variable y corresponden a los estudios de correlación, sin embargo, las hipótesis de tipo correlacional no sólo establecen el vínculo entre más de una variable, sino también la manera en que están asociadas. En tal sentido, las hipótesis planteadas por los investigadores son las siguientes:

Hipótesis general:

Las habilidades blandas impactan en la gestión de la mezcla de marketing de las empresas del Emporio Comercial Gamarra, Perú.

Hipótesis específicas:

El liderazgo impacta en la gestión del producto de las empresas del Emporio Comercial Gamarra, distrito de La Victoria, Perú. 
El liderazgo impacta en la gestión del precio de las empresas del Emporio Comercial Gamarra, distrito de La Victoria, Perú.

El liderazgo impacta en la gestión de la plaza de las empresas del Emporio Comercial Gamarra, Perú.

El liderazgo impacta en la gestión de la promoción de las empresas del Emporio Comercial Gamarra, Perú.

Las habilidades de comunicación impactan en la gestión del producto de las empresas del Emporio Comercial Gamarra, Perú.

Las habilidades de comunicación impactan en la gestión del precio de las empresas del Emporio Comercial Gamarra, Perú.

Las habilidades de comunicación impactan en la gestión de la plaza de las empresas del Emporio Comercial Gamarra, Perú.

Las habilidades de comunicación impactan en la gestión de la promoción de las empresas del Emporio Comercial Gamarra, Perú.

En cuanto a los objetivos de la investigación, que de acuerdo con Bernal (2010) se formulan con la meta de describir la finalidad de la investigación y toda esta se orientará a lograr su cumplimiento, es preciso mencionar que son:

Objetivo general.

Determinar en qué medida las habilidades blandas impactan en la gestión de la mezcla de marketing de las empresas del Emporio Comercial Gamarra, Perú.

Objetivos específicos.

Determinar en qué medida el liderazgo impacta en la gestión del producto de las empresas del Emporio Comercial Gamarra, Perú.

Determinar en qué medida el liderazgo impacta en la gestión del precio de las empresas del Emporio Comercial Gamarra, Perú.

Determinar en qué medida el liderazgo impacta en la gestión de la plaza de las empresas del Emporio Comercial Gamarra, Perú.

Determinar en qué medida el liderazgo impacta en la gestión de la promoción de las empresas del Emporio Comercial Gamarra, Perú. 
Determinar en qué medida las habilidades de comunicación impactan en la gestión del producto de las empresas del Emporio Comercial Gamarra, Perú.

Determinar en qué medida las habilidades de comunicación impactan en la gestión del precio de las empresas del Emporio Comercial Gamarra, Perú.

Determinar en qué medida las habilidades de comunicación impactan en la gestión de la plaza de las empresas del Emporio Comercial Gamarra, Perú.

Determinar en qué medida las habilidades de comunicación impactan en la gestión de la promoción de las empresas del Emporio Comercial Gamarra, Perú.

En cuanto a la justificación de la investigación, se debe resaltar que ésta denota gran importancia debido a que podrá ser utilizado para la ampliación de previas teorías o modelos de carácter teórico relacionados a las habilidades blandas en el mundo empresarial, teniendo en cuenta que son muy escasos los estudios que relacionan dichas habilidades con la mezcla de marketing en las empresas, por ende, este estudio va a permitir la realización de una innovación científica; además la metodología presente en este estudio podrá ser utilizado para posteriores investigaciones de similar carácter, con la finalidad de beneficiar a la comunidad científica interesada en las ciencias empresariales.

\section{REVISIÓN TEÓRICA}

\section{Habilidades blandas.}

Según Ortega (2017, p. 7), las habilidades blandas son "un conjunto de destrezas que permiten desempeñarse mejor en las relaciones laborales y personales". Asimismo, sostiene Perreault (2004), las habilidades blandas son un conjunto de atributos o cualidades de una persona que le permiten distinguirse de las demás a pesar de que éstas puedan poseer similares habilidades tipo de experiencia.

De acuerdo a Davies, Fidler \& Gorbis (2011), en un estudio elaborado para el Instituto de Investigación de la Universidad de Phoenix, en Estados Unidos, las habilidades blandas del personal que componga cualquier organización serán generadoras de ventajas competitivas que permitirán alcanzar el éxito en el año 2020.

Ortega (2017, p. 7) a su vez sostiene que "los especialistas coinciden en que las habilidades técnicas se pueden enseñar mucho más fácilmente que las habilidades 
blandas", por lo que se puede inferir que la prioridad de retención de trabajadores en las organizaciones se da hacia aquellos individuos que desarrollen y muestren habilidades blandas orientadas al mundo empresarial.

Entre las habilidades blandas esenciales en el mundo administrativo se tienen, según Madrigal (2009):
a) Inteligencia emocional.
b) Habilidades de comunicación.
c) Administración del tiempo.
d) Creatividad.
e) Capacidad de toma de decisiones.
f) Trabajo en equipo y manejo de conflictos.
g) Liderazgo.
h) Poder de negociación.
i) Poder de motivación.
j) Formulación de estrategias.
k) Capacidad de pensamiento.

No cabe duda, que las habilidades blandas son un tema con mucho por explorar e investigar, en especial por el impacto que generan en el mundo de los negocios.

\section{Mezcla de marketing.}

Kotler (2003, p. 90) presenta la definición de mezcla de marketing como "una serie de herramientas que se utilizan para influir en las ventas". El concepto de mezcla de marketing ha variado significativamente a lo largo de los años y ha sido susceptible a ser cambiado a lo largo de los años, sin embargo, normalmente se toma como referencia el modelo original de las 4P establecido por McCarthy.

Cabe acortar que la mezcla de marketing de una empresa debe estar interrelacionada de forma integrada para que funcione y es un reto difícil de afrontar para muchas empresas, ya que usualmente la responsabilidad de los diferentes elementos de la mezcla del marketing está en manos de diferentes individuos o departamentos (Kotler, 2003). Las 4P de la mezcla de marketing corresponden al producto, el precio, la plaza y la distribución.

En cuanto al producto, de acuerdo con Staton, Etzel y Walker (2007) este está conformado por una serie de atributos o cualidades, que pueden ser tanto tangibles como intangibles y que abarcan características propias del mismo. Las partes que las 
conforman incluyen empaques, el envase y embalaje, colores, calidad y marca, además de otras tales como el servicio y la reputación del vendedor. Cabe resaltar que el producto puede ser un bien intercambiado, un servicio prestado, un lugar visitado, una persona o inclusive una idea formulada. Los clientes, por lo tanto, continúan Staton et al. (2007) no compran tan solo un bien o servicio, motivados por actividades de marketing, sino que compran un conjunto de atributos mencionados líneas arriba, además de la obtención de determinada satisfacción a manera de beneficios que esperan recibir de dicho producto.

El precio "es la cantidad de dinero u otros elementos de utilidad que se necesitan para adquirir un producto" (Staton et al., 2007, p. 338). Este precio podría subir o bajar de acuerdo a diversas políticas que pueda utilizar la empresa, generalmente relacionadas a decisiones financieras (Fernández, 2018).

La plaza es definida por la Real Academia Española (2017), como un espacio, sitio o lugar donde se venden artículos; en otras palabras, la ubicación del producto en puntos de ventas propios o ajenos. La accesibilidad, o mejor dicho, facilidad con la cual el cliente adquirirá los productos dependerá en gran medida al desarrollo de la plaza.

En cuanto a la promoción Hernández y Maubert (2012), plantean que el precio tiene como característica principal el de la comunicación con individuos, grupos $u$ organizaciones, sea el tipo que sea, con el fin la realización de intercambios mediante la información y posterior persuasión para con los productos. Asimismo, cabe acotar que los objetivos para una correcta utilización de la promoción son el de crear conocimiento, estimular la demanda, identificar clientes potenciales y conservar los leales, entre otros (Hernández y Maubert, 2012).

Cada empresa exitosa deberá contar con un grupo humano encargado de la gestión de las 4P del marketing previamente descritas, estas personas estarán a cargo de determinar diferentes políticas de marketing con la finalidad de subsistir o mantener el éxito en el mercado (Mullins, Walker, Boyd y Larréché, 2007). Así se debe delimitar las siguientes políticas empresariales relacionadas a las 4P de la mezcla de marketing:

Políticas de producto:

a) Gestión de la amplitud de la línea del producto.

b) Gestión del refinamiento técnico del producto.

c) Gestión de la calidad del producto.

d) Gestión de la calidad de servicio. 
Políticas de precio:

a) Gestión de los niveles de precios al por mayor.

b) Gestión de los niveles de precios al por menor.

Políticas de plaza o distribución:

a) Gestión del grado de integración vertical directa.

b) Gestión del gasto de promoción comercial como porcentaje de ventas.

c) Gestión de la delimitación geográfica del mercado.

Políticas de promoción

a) Gestión de gastos de publicidad como porcentaje de ventas.

b) Gestión de promociones de ventas como porcentaje de ventas.

c) Gestión de los gastos de los vendedores como porcentaje de ventas.

Los cuatro elementos de las 4P de la mezcla de marketing guardan relación entre sí mismo y por consiguiente las decisiones de un área afectan las acciones de otra (Staton et al, 2007). Por ejemplo, si se decide aumentar la calidad del producto, indudablemente se deberá incurrir en un aumento de los costos y por ende del precio, lo cual podrá no ser aceptado por la totalidad de clientes, por lo que se deberá analizar en qué plaza será viable el cambio, y en cuáles será necesario mayor promoción.

\section{MATERIALES Y MÉTODOS}

\section{Diseño de Investigación.}

Para Hernández Sampieri et al., (2014) el diseño de la investigación es similar a la estrategia desarrollada con el fin de obtener información en forma de datos, requerida en este trabajo investigativo, y cuyo fin es la búsqueda de la respuesta al planteamiento previamente hecho; ante esto, a líneas debajo se procederá a detallar el enfoque, el nivel y el diseño de este estudio:

El enfoque de la investigación es cuantitativo. Para Bernal (2010), todo enfoque cuantitativo mide características de ciertos fenómenos sociales observado por los investigadores, y que para ello será necesario detallar un marco teórico pertinente al problema a analizar, a fin de obtener una amplia lista de dimensiones que expresen determinadas relaciones entre las variables a estudiar. 
Esta investigación presenta la característica de ser de nivel correlativo-causal, ya que busca demostrar relaciones entre dos o más variables en determinado momento, de manera correlacional, o denotando una relación de causa y efecto (Hernández Sampieri et al., 2014).

Este estudio es de diseño no experimental, debido a que no se realizó ninguna manipulación deliberada de alguna de las variables y los fenómenos fueron estudiados en su entorno natural para su correspondiente análisis (Hernández Sampieri et al., 2014).

\section{Población y muestra.}

Una población es la cantidad total de individuos en los cuáles se basa el objeto del (Del Cid, Méndez y Sandoval, 2011). Para esta investigación, la población está conformada por la totalidad de trabajadores que realizan actividades remuneradas en el Emporio Comercial Gamarra, siendo en total 40928 individuos, de acuerdo al último informe presentado por el Instituto Nacional de Estadística e Informática de Perú (INEI, 2017, p.64).

La muestra representa a la población, ésta es calculada a través de la estadística tomando en consideración el nivel de confianza deseado y el porcentaje de error máximo.

Para una población de 48928 individuos, la muestra será 381, teniendo como condicionales un nivel de confianza de $95 \%$, con un $5 \%$ de error máximo y $50 \%$ de probabilidad tanto a favor como en contra, tal y como se muestra en la siguiente fórmula:

$$
\begin{gathered}
n=\frac{\left(\sigma^{2} * N * p * q\right)}{\left(\left(e^{2} *(N-1)\right)+\left(\sigma^{2} * p * q\right)\right)} ; \frac{\left(1,96^{2} * 40928 * 0,5 * 0,5\right)}{\left(\left(0,05^{2} *(40928-1)\right)+\left(1,96^{2} * 0,5 * 0,5\right)\right)} \\
=381
\end{gathered}
$$

Se utilizó el criterio aleatorio para la aplicación del instrumento. Los datos se obtuvieron en la misma zona de estudio (Emporio Comercial Gamarra) en el mes de agosto del 2018, esta operación estuvo a cargo de un equipo conformado por 8 personas, quienes concretaron citas para obtener la información requerida, la cual fue posteriormente reportada al investigador principal con una frecuencia semanal.

\section{Técnicas e instrumentos de recolección de datos.}

Es conocido que toda actividad investigativa requiere de la recolección de información relevante relacionada al fenómeno estudio. Para Hernández Sampieri et al., (2014, p. 
217), "en fenómenos sociales, el instrumento más utilizado para recolectar los datos es el cuestionario". Un cuestionario consta de una serie de preguntas colocadas a propósito, con la finalidad de integrar una o más variables a medir con la estadística.

El cuestionario, será aplicado a la muestra, esto eso, a 381 trabajadores que realizan actividades en el Emporio Comercial Gamarra, en el distrito de La Victoria, Lima, Perú.

El cuestionario consta de 15 preguntas, relacionadas a los indicadores a evaluar de acuerdo a las dimensiones relacionadas a las variables de la investigación, de acuerdo a lo mostrado en la figura 2.

Figura 2. Distribución de preguntas aplicadas en cuestionario.

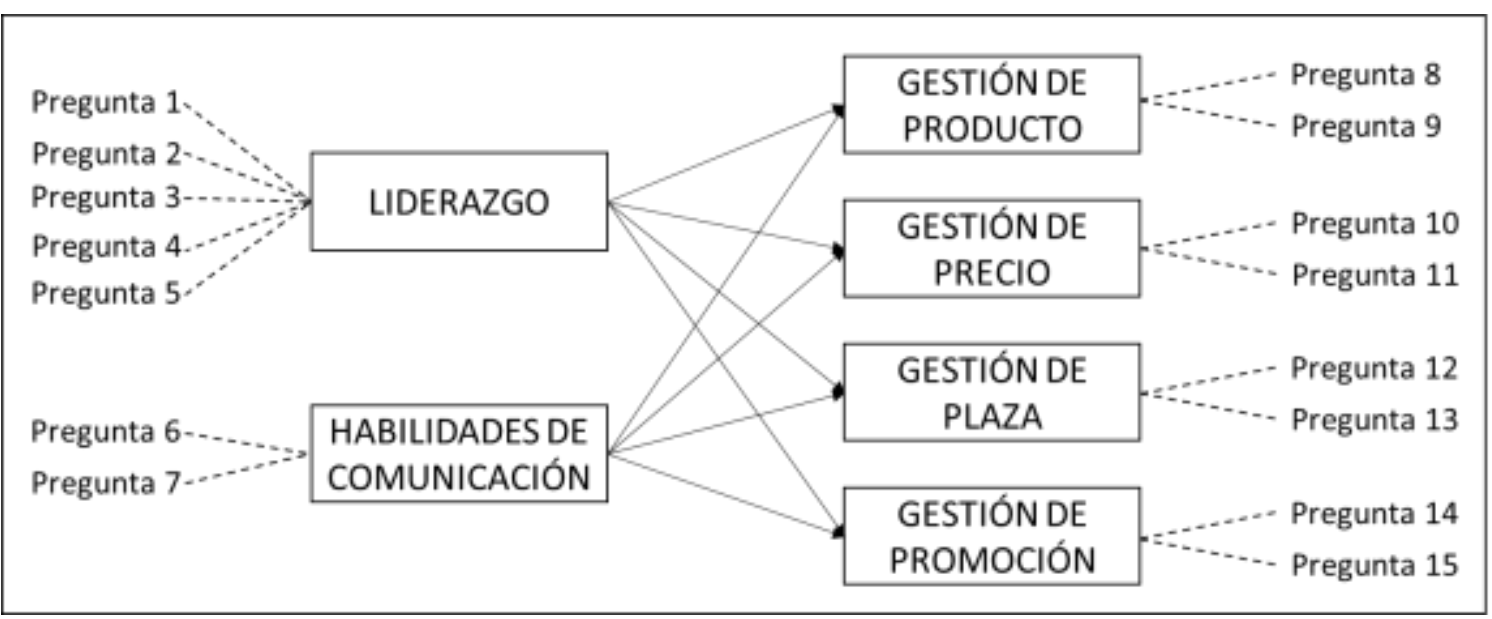

Fuente: Elaboración propia.

\section{Procedimientos de recolección de datos y presentación de resultados.}

Para la recolección de datos y presentación de sus resultados, los investigadores dividieron la investigación en siete etapas, siendo estas:

Etapa 1: selección e identificación de la muestra, que de acuerdo a lo calculado estadísticamente, ascienden a 381 trabajadores de Gamarra.

Etapa 2: delimitación del lugar en el cual los datos serán recolectados, el cual es el Emporio Comercial Gamarra, delimitado por las avenidas Aviación, México y 28 de Julio del distrito de la Victoria, distrito de Lima, en Perú.

Etapa 3: aplicación del instrumento cuestionario a la muestra descrita en la etapa 1, cabe recalcar que este cuestionario contiene 15 preguntas. Es preciso indicar que las alternativas de respuestas son de tipo Lickert de 5 niveles. 
Etapa 4: digitación y tabulación de los resultados obtenidos del cuestionario en el programa Microsoft Excel.

Etapa 5: importación de los datos inicialmente digitados Microsoft Excel, en el software estadístico SPSS.

Etapa 6: realización de análisis probabilísticos y tablas, con la finalidad de dar mayor visibilidad a los resultados obtenidos.

Etapa 7: Emisión de conclusiones y recomendaciones, teniendo como base lo avanzado en las seis primeras etapas.

\section{Pruebas de fiabilidad del instrumento.}

A fin de cuantificar la fiabilidad del instrumento a aplicar, se realizaron las pruebas de Alfa de Cronbach, el coeficiente de Spearman-Brown y el coeficiente de dos mitades de Guttman.

Tabla 1. Resumen del procesamiento de casos, pruebas de fiabilidad del instrumento.

\begin{tabular}{llrr}
\hline & & $\mathrm{N}$ & \multicolumn{1}{c}{$\%$} \\
\hline \multirow{3}{*}{ Casos } & Válido & 381 & 100,0 \\
& Excluido $^{\mathrm{a}}$ & 0 &, 0 \\
& Total & 381 & 100,0 \\
\hline
\end{tabular}

a. La eliminación por lista se basa en todas las variables del procedimiento.

Fuente: Elaboración propia.

Como se puede apreciar en la tabla 1, todos los resultados obtenidos a través del cuestionario fueron considerados válidos, esto es un $100 \%$.

Tabla 2. Estadísticas de fiabilidad del instrumento.

\begin{tabular}{lllr}
\hline & Parte 1 & Valor & 0,877 \\
Alfa de Cronbach & N de elementos & $7^{\text {a }}$ \\
& & Valor & 0,844 \\
& Parte 2 & N de elementos & $8^{\text {b }}$ \\
& $N$ total de elementos & 15 \\
\hline Correlación entre formularios & & 0,957
\end{tabular}




\begin{tabular}{llr}
\hline $\begin{array}{lr}\text { Coeficiente de Spearman- } \\
\text { Brown }\end{array}$ & Longitud igual & 0,968 \\
& Longitud desigual & 0,978 \\
\hline \hline Coeficiente de dos mitades de Guttman &, 971 \\
\hline \hline
\end{tabular}

Fuente: Elaboración propia.

Según la tabla 2, los resultados estadísticos de fiabilidad del instrumento nos muestran que el valor de Alfa de Cronbach es de 0,877 para la parte 1 y 0,844 para la parte dos, la correlación entre formularios es de 0,957, el coeficiente de Spearman-Brown es de 0,968 longitud igual y de 0,978 longitud desigual, además de que el coeficiente de dos mitades de Guttman es de 0,971 . Ante estos resultados, los cuales son muy cercanos a 1 , se puede afirmar que el cuestionario aplicado tiene un muy alto nivel de fiabilidad, de acuerdo con los estándares generalmente aceptados.

Cabe resaltar, que el instrumento a utilizar fue validado a través del juicio de expertos, conformada por una terna de tres docentes investigadores con grado de doctor en ciencias empresariales, todos ellos dieron opiniones favorables y por ende garantizando su validez.

\section{ANÁLISIS DE RESULTADOS}

A fin de confirmar o rechazar las hipótesis planteadas, se procedió a aplicar la herramienta estadística de Chi-cuadrado de Pearson, la cual es una prueba estadística descriptiva que muestra asociación o no de variables, de acuerdo al valor dado de significación asintótica bilateral, la cual deberá ser menor a 0,05 para un nivel de confianza del $95 \%$. A continuación, se detallará el análisis de los resultados obtenidos para cada prueba de hipótesis:

Tabla 3. Pruebas de Chi-cuadrado: las habilidades blandas impactan en la gestión de la mezcla de marketing de las empresas del Emporio Comercial Gamarra, Perú.

\begin{tabular}{lrrr} 
& \multicolumn{1}{c}{ Valor } & gl & \multicolumn{2}{c}{$\begin{array}{c}\text { Sig. asintótica } \\
\text { (bilateral) }\end{array}$} \\
\hline Chi-cuadrado de Pearson & $185,142^{\text {a }}$ & 45 & 0,000 \\
$\begin{array}{l}\text { Razón de verosimilitud } \\
\text { Asociación lineal por }\end{array}$ & 123,337 & 45 & 0,000 \\
lineal & 43,989 & 1 & 0,000
\end{tabular}


Fuente: Elaboración propia.

De acuerdo a lo mostrado en la tabla 3, la prueba de Chi-cuadrado de Pearson dio como resultado el valor de 185,142, con grados de liberad de 45 y significación asintótica bilateral de 0,000, y, siendo este último valor menor a 0,05, nos permite afirmar que existe asociación entre las variables estudiadas y por ende aceptar la hipótesis planteada, por lo tanto, se acepta la hipótesis general: las habilidades blandas impactan en la gestión de la mezcla de marketing de las empresas del Emporio Comercial Gamarra, Perú.

Tabla 4. Pruebas de Chi-cuadrado: El liderazgo impacta en la gestión del producto de las empresas del Emporio Comercial Gamarra, distrito de La Victoria, Perú.

\begin{tabular}{lrrr} 
& \multicolumn{1}{c}{ Valor } & gl & \multicolumn{2}{c}{$\begin{array}{c}\text { Sig. asintótica } \\
\text { (bilateral) }\end{array}$} \\
\hline Chi-cuadrado de Pearson & $161,157^{\mathrm{a}}$ & 70 & 0,000 \\
Razón de verosimilitud & 139,160 & 70 & 0,000 \\
$\begin{array}{lrrc}\text { Asociación lineal por } \\
\text { lineal }\end{array}$ & 15,664 & 1 & 0,017 \\
\hline N de casos válidos & 381 & & \\
\hline \hline
\end{tabular}

Fuente: Elaboración propia.

Según tabla 4, la prueba de Chi-cuadrado de Pearson dio como resultado el valor de 185,142, con grados de liberad de 45 y significación asintótica bilateral de 0,000, y, siendo este último valor menor a 0,05. Esto nos permite afirmar que existe asociación entre las variables estudiadas y por ende aceptar la hipótesis específica planteada: el liderazgo impacta en la gestión del producto de las empresas del Emporio Comercial Gamarra, distrito de La Victoria, Perú.

Tabla 5. Pruebas de Chi-cuadrado: El liderazgo impacta en la gestión del precio de las empresas del Emporio Comercial Gamarra, distrito de La Victoria, Perú.

\begin{tabular}{lrrr} 
& \multicolumn{1}{c}{ Valor } & gl & \multicolumn{2}{c}{$\begin{array}{c}\text { Sig. asintótica } \\
\text { (bilateral) }\end{array}$} \\
\hline Chi-cuadrado de Pearson & $175,152^{\mathrm{a}}$ & 55 & 0,000 \\
$\begin{array}{l}\text { Razón de verosimilitud } \\
\text { Asociación lineal por }\end{array}$ & 133,437 & 55 & 0,000 \\
lineal & 53,989 & 1 & 0,001
\end{tabular}


Fuente: Elaboración propia.

En la tabla 5 se puede ver que, la prueba de Chi-cuadrado de Pearson dio como resultado el valor de 175,152, con grados de liberad de 55 y significación asintótica bilateral de 0,000, y, siendo este último valor menor a 0,05, nos permite afirmar que existe asociación entre las variables estudiadas y por lo tanto se afirma la hipótesis específica el liderazgo impacta en la gestión del precio de las empresas del Emporio Comercial Gamarra, distrito de La Victoria, Perú.

Tabla 6. Pruebas de Chi-cuadrado: El liderazgo impacta en la gestión de la plaza de las empresas del Emporio Comercial Gamarra, distrito de La Victoria, Perú.

\begin{tabular}{lrrr} 
& \multicolumn{1}{c}{ Valor } & gl & \multicolumn{2}{c}{$\begin{array}{c}\text { Sig. asintótica } \\
\text { (bilateral) }\end{array}$} \\
\hline Chi-cuadrado de Pearson & $185,142^{\text {a }}$ & 45 & 0,000 \\
Razón de verosimilitud & 123,337 & 45 & 0,000 \\
$\begin{array}{l}\text { Asociación lineal por } \\
\text { lineal }\end{array}$ & 33,989 & 1 & 0,002 \\
\hline N de casos válidos & 381 & & \\
\hline \hline
\end{tabular}

Fuente: Elaboración propia.

De acuerdo con el análisis expuesto en la tabla 6, la prueba de Chi-cuadrado de Pearson dio como resultado el valor de 185,142, con grados de liberad de 45 y significación asintótica bilateral de $0,000, y$, siendo este último valor menor a 0,05 , nos permite afirmar que existe asociación entre las variables estudiadas y por ende aceptar la hipótesis específica: el liderazgo impacta en la gestión de la plaza de las empresas del Emporio Comercial Gamarra, distrito de La Victoria, Perú.

Tabla 7. Pruebas de Chi-cuadrado: El liderazgo impacta en la gestión de la promoción de las empresas del Emporio Comercial Gamarra, distrito de La Victoria, Perú.

\begin{tabular}{lrrr} 
& \multicolumn{1}{c}{ Valor } & gl & \multicolumn{2}{c}{$\begin{array}{c}\text { Sig. asintótica } \\
\text { (bilateral) }\end{array}$} \\
\hline Chi-cuadrado de Pearson & $188,142^{\mathrm{a}}$ & 46 & 0,001 \\
Razón de verosimilitud & 126,337 & 46 & 0,000 \\
$\begin{array}{l}\text { Asociación lineal por } \\
\text { lineal }\end{array}$ & 33,989 & 1 & 0,002 \\
\hline N de casos válidos & 381 & & \\
\hline \hline
\end{tabular}


Fuente: Elaboración propia.

Para la tabla 7, la prueba de Chi-cuadrado de Pearson dio como resultado el valor de 188,142, con grados de liberad de 46 y significación asintótica bilateral de 0,001, y, siendo este último valor menor a 0,05, nos permite afirmar que existe asociación entre las variables estudiadas y por ende aceptar la hipótesis específica: el liderazgo impacta en la gestión de la promoción de las empresas del Emporio Comercial Gamarra, distrito de La Victoria, Perú.

Tabla 8. Pruebas de Chi-cuadrado: Las habilidades de comunicación impactan en la gestión del producto de las empresas del Emporio Comercial Gamarra, Perú.

\begin{tabular}{lrrr} 
& \multicolumn{1}{c}{ Valor } & gl & \multicolumn{1}{c}{$\begin{array}{c}\text { Sig. asintótica } \\
\text { (bilateral) }\end{array}$} \\
\hline Chi-cuadrado de Pearson & $191,142^{\mathrm{a}}$ & 47 & 0,002 \\
Razón de verosimilitud & 129,337 & 47 & 0,000 \\
$\begin{array}{l}\text { Asociación lineal por } \\
\text { lineal }\end{array}$ & 33,989 & 1 & 0,002 \\
\hline N de casos válidos & 381 & & \\
\hline \hline
\end{tabular}

Fuente: Elaboración propia.

En la tabla 8, la prueba de Chi-cuadrado de Pearson dio como resultado el valor de 191,142, con grados de liberad de 47 y significación asintótica bilateral de 0,002, y, siendo este último valor menor a 0,05, permite afirmar que existe asociación entre las variables estudiadas y por ende aceptar la hipótesis específica previamente planteada: Las habilidades de comunicación impactan en la gestión del producto de las empresas del Emporio Comercial Gamarra, Perú.

Tabla 9. Pruebas de Chi-cuadrado: Las habilidades de comunicación impactan en la gestión del precio de las empresas del Emporio Comercial Gamarra, Perú.

\begin{tabular}{lrrr} 
& \multicolumn{1}{c}{ Valor } & gl & \multicolumn{2}{c}{$\begin{array}{c}\text { Sig. asintótica } \\
\text { (bilateral) }\end{array}$} \\
\hline Chi-cuadrado de Pearson & $185,142^{\mathrm{a}}$ & 45 & 0,000 \\
Razón de verosimilitud & 123,337 & 45 & 0,000 \\
$\begin{array}{l}\text { Asociación lineal por } \\
\text { lineal }\end{array}$ & 33,989 & 1 & 0,002 \\
\hline N de casos válidos & 381 & & \\
\hline \hline
\end{tabular}

Fuente: Elaboración propia. 
De acuerdo a lo visto en la tabla 9, la prueba de Chi-cuadrado de Pearson dio como resultado el valor de 185,142, con grados de liberad de 45 y significación asintótica bilateral de 0,000, y, siendo este último valor menor a 0,05, nos permite afirmar que existe asociación entre las variables estudiadas y por ende aceptar la hipótesis específica planteada: Las habilidades de comunicación impactan en la gestión del precio de las empresas del Emporio Comercial Gamarra, Perú.

Tabla 10. Pruebas de Chi-cuadrado: Las habilidades de comunicación impactan en la gestión de la plaza de las empresas del Emporio Comercial Gamarra, Perú.

\begin{tabular}{lrrr} 
& \multicolumn{1}{c}{ Valor } & gl & \multicolumn{2}{c}{$\begin{array}{c}\text { Sig. asintótica } \\
\text { (bilateral) }\end{array}$} \\
\hline Chi-cuadrado de Pearson & $196,142^{\mathrm{a}}$ & 45 & 0,000 \\
Razón de verosimilitud & 133,337 & 45 & 0,000 \\
Asociación lineal por & 27,989 & 1 & 0,002 \\
lineal & 381 & & \\
\hline N de casos válidos &
\end{tabular}

Fuente: Elaboración propia.

Según la tabla 10, la prueba de Chi-cuadrado de Pearson dio como resultado el valor de 196,142, con grados de liberad de 45 y significación asintótica bilateral de 0,000, y, siendo este último valor menor a 0,05, nos permite afirmar que existe asociación entre las variables estudiadas y por ende aceptar la hipótesis específica: Las habilidades de comunicación impactan en la gestión de la plaza de las empresas del Emporio Comercial Gamarra, Perú.

Tabla 11. Pruebas de Chi-cuadrado: Las habilidades de comunicación impactan en la gestión de la promoción de las empresas del Emporio Comercial Gamarra, Perú.

\begin{tabular}{lrrr} 
& \multicolumn{1}{c}{ Valor } & gl & \multicolumn{2}{c}{$\begin{array}{c}\text { Sig. asintótica } \\
\text { (bilateral) }\end{array}$} \\
\hline Chi-cuadrado de Pearson & $199,142^{\mathrm{a}}$ & 55 & 0,000 \\
Razón de verosimilitud & 163,357 & 55 & 0,000 \\
Asociación lineal por & 27,889 & 1 & 0,002 \\
lineal & 381 & & \\
\hline N de casos válidos &
\end{tabular}

Fuente: Elaboración propia.

Por último, y de acuerdo con la tabla 11, la prueba de Chi-cuadrado de Pearson dio como resultado el valor de 199,142, con grados de liberad de 55 y significación asintótica 
bilateral de 0,000, y, siendo este último valor menor a 0,05 , nos permite afirmar que existe asociación entre las variables estudiadas y por ende aceptar la hipótesis específica: Las habilidades de comunicación impactan en la gestión de la promoción de las empresas del Emporio Comercial Gamarra, Perú.

\section{DISCUSIÓN Y CONCLUSIONES}

Queda demostrado que las habilidades blandas impactan positivamente en la gestión de la mezcla de marketing, de acuerdo a este estudio realizado en el emporio comercial Gamarra, ubicado en La Victoria, Lima, Perú.

Gracias al análisis estadístico aplicada en la información recolectada, se pudo comprobar la hipótesis general, esto es, que las habilidades blandas impactan en la gestión de la mezcla de marketing de las empresas del Emporio Comercial Gamarra, Perú. Se comprueba que la mezcla de mercadotécnica está asociado a la generación de ventajas competitivas, tal y como lo menciona Staton (2007), asimismo, las habilidades blandas mostradas por los trabajadores también se verán traducidas en la generación de ventajas competitivas, lo cual refuerza lo planteado por Davies, Fidler \& Gorbis (2011). Será conveniente, entonces, realizar capacitaciones a los trabajadores enfocados al adiestramiento no solo de habilidades técnicas, sino también las blandas, las cuales son de difícil aprendizaje, según lo mencionado por Ortega (2017), pero que impactan positivamente en la gestión de la mezcla de marketing.

La estadística también permitió dar validez a las hipótesis específicas planteadas, las cuales son:

El liderazgo impacta en la gestión del producto de las empresas del Emporio Comercial Gamarra, distrito de La Victoria, Perú.

El liderazgo impacta en la gestión del precio de las empresas del Emporio Comercial Gamarra, distrito de La Victoria, Perú.

El liderazgo impacta en la gestión de la plaza de las empresas del Emporio Comercial Gamarra, Perú.

El liderazgo impacta en la gestión de la promoción de las empresas del Emporio Comercial Gamarra, Perú. 
Las habilidades de comunicación impactan en la gestión del producto de las empresas del Emporio Comercial Gamarra, Perú.

Las habilidades de comunicación impactan en la gestión del precio de las empresas del Emporio Comercial Gamarra, Perú.

Las habilidades de comunicación impactan en la gestión de la plaza de las empresas del Emporio Comercial Gamarra, Perú.

Las habilidades de comunicación impactan en la gestión de la promoción de las empresas del Emporio Comercial Gamarra, Perú.

Se invita a otros investigadores a elaborar investigaciones similares en otras áreas geográficas, pudiendo utilizar los mismos materiales y métodos expuestos en el presente trabajo, a fin de generar mayor discusión en este tema. Asimismo, se invita a la comunidad científica a investigar aún más sobre las habilidades blandas y su impacto en las diferentes corrientes relacionadas a la actividad empresarial, sean relacionados al marketing, al desarrollo organizacional, a la atención al cliente y ventas, entre otras.

\section{REFERENCIAS BIBLIOGRÁFICAS}

Ascón, J. E., García, M. y Pedraza, C. I. (2018). Las habilidades directivas en las instituciones de educación superior, diseño estratégico con enfoque de liderazgo. Revista Científica ECOCIENCIA, 5(2). Recuperado de http://ecociencia.ecotec.edu.ec/upload/php/files/abril18/05.pdf

Bernal, C. (2010). Metodología de la investigación. (3.a ed.). Bogotá, Colombia: Pearson Educación.

Davies, A., Fidler, D. \& Gorbis, M. (2011). Future Work Skills 2020. (1.a ed.). Arizona, Estados Unidos: University of Phoenix Research Institute.

Del Cid, A., Méndez, R. y Sandoval, F. (2011). Investigación. Fundamentos y metodología. (2.a ed.). Naucalpan de Juárez, México: Pearson Educación.

Fernández, V. H. (2018). Punto de equilibrio y su incidencia en las decisiones financieras de empresas editoras en Lima. QUIPUKAMAYOC, 26(52). DOI: http://dx.doi.org/10.15381/quipu.v26i52.15507

Hernández, C., y Maubert, V. (2012). Mercadotecnia. (1.a ed.). Naucalpan de Juárez, México: Pearson Educación. 
Hernández Sampieri, R., Fernández, C., y Baptista, P. (2014). Metodología de la investigación. (6.a ed.). México D.F., México: McGraw-Hill.

INEI. (2017). Características de las empresas del Emporio Comercial Gamarra, 2016. Recuperado de https://www.inei.gob.pe/media/MenuRecursivo/publicaciones_digitales/Est/Lib14 63/libro.pdf

Jackson, G., \& Ahuja, V. (2016). Dawn of the digital age and the evolution of the marketing mix. Journal of Direct, Data and Digital Marketing Practice, 17(3), 170186. Recuperado de http://dx.doi.org/10.1057/dddmp.2016.3

Gallardo, Y. y Moreno, A. (1999). Módulo 3: Recolección de datos - Serie aprendiendo a investigar. (3.a ed.). Bogotá, Colombia: ICFES.

Kotler, P. (2003). Los 80 conceptos esenciales del Marketing de la A a la Z. (1.a ed.). Madrid, España: Pearson Educación.

Madrigal, B. (2007). Habilidades directivas. (2.a ed.). México D.F., México: McGraw-Hill.

Marrero, O., Mohamed, R. y Xifra, J. (2018). Habilidades blandas: necesarias para la formación integral del estudiante universitario. Revista Científica ECOCIENCIA, edición 2018. Recial de http://ecociencia.ecotec.edu.ec/upload/php/files/diciembreespe/04.pdf

Minaya, M. y Fernández, V. H. (2018). Implementación del sistema de costeo ABC y la percepción de la mejora continua en empresas industriales de metal mecánica en Lurigancho, Lima. Año 2017. UCV - Scientia, 10(1). DOI: dx.doi.org/10.18050/RevUcv-Scientia.v10n1a3

Mullins, J., Walker, O., Boyd, H. y Larréché, J.C. (2007). Administración del marketing. (5.a ed.). México D.F., México: McGraw-Hill.

Ortega, C. E. (2017). Desarrollo de habilidades blandas desde edades tempranas. (1.a ed.). Guayaquil, Ecuador: Fondo Editorial Universidad ECOTEC.

Ortega, C. E., Febles, J. P. y Estrada, V. (2016). Fundamentación teórico-metodológica de una estrategia para desarrollar habilidades blandas desde la enseñanza inicial. Revista Científica ECOCIENCIA, 3(3). Recuperado de http://ecociencia.ecotec.edu.ec/upload/php/files/junio/1.pdf 
Patrone, P. (2 de enero de 2018). The Skills Companies Need Most in 2018 - And The Courses to Get Them [Mensaje en un blog]. Recuperado de https://www.linkedin.com/pulse/skills-companies-need-most-2018-courses-getthem-paul-petrone

Perreault, H. (2004). Business educators can take a leadership role in character education. Business Education Forum, 1(59), 43-53

Plomaritou, E. I. (2008). A proposed application of the marketing mix concept to tramp \& liner shipping companies. Management: Journal of Contemporary Management Issues, 13(1), 59-71. Recuperado de https://search.proquest.com/docview/221192336

Real Academia Española. (2001). Diccionario de la lengua española. (23.a ed.). Consultado en http://dle.rae.es/index.html

Staton, W., Etzel, M. y Walker, B. (2007). Fundamentos de Marketing. (14.a ed.). México D.F., México: McGraw-Hill.

Zermeño, A. M. y Lozano, A. (2016). Desarrollo de competencias interpersonales en ambientes virtuales. CPU-e, Revista de Investigación Educativa, 22(1). Recuperado de https://www.redalyc.org/pdf/2831/283143550009.pdf 